\title{
Reduced levels of p15INK4b, p16INK4a, p21cip1 and p27kip1 in pancreatic carcinoma
}

\author{
GANG LI $^{1}$, YUAN JI ${ }^{2}$, CHEN LIU ${ }^{3}$, JINGQI LI ${ }^{3}$ and YINGQI ZHOU ${ }^{1}$ \\ ${ }^{1}$ The Third General Surgery Department, Zhanghai Hospital, Second Military Medical University; \\ ${ }^{2}$ Department of Pathology, Zhongshan Hospital, Fudan University; ${ }^{3}$ Laboratory of Cancer Research, \\ Tongji University School of Medicine, Shanghai, P.R. China
}

Received November 1, 2011; Accepted January 10, 2012

DOI: $10.3892 / \mathrm{mmr} .2012 .771$

\begin{abstract}
Pancreatic carcinoma is one of the leading causes of cancer mortality worldwide, although the molecular mechanisms of this disease are poorly understood. The aim of this study was to examine the expression of cyclin-dependent kinase inhibitors (CDKIs) and the epigenetic modifications in the promoters of these genes. We also evaluated the correlation between the methylation status of $C D K I$ genes and smoking habit in clinical pancreatic carcinoma specimens. Western blotting and real-time PCR were performed to assess CDKI expression. Methylation-specific PCR was carried out to examine the methylation status of the promoters of $C D K I$ genes. In this study, we revealed that reduced levels of the CDKI proteins, p15INK4b, p16INK4a, p21cip1 and p27kip1, are a prominent feature of pancreatic carcinoma patients. The DNA hypermethylation of the promoter was observed in $40 \%$ ( 2 of 5) of the p15INK $4 b$ genes, $60 \%$ ( 3 of 5) of the pl6INK4a genes and $60 \%$ of the p21cipl genes, which markedly correlated with their decreased mRNA expression. No hypermethylation was detected in the p27kipl gene promoter in 5 pancreatic carcinoma patients with markedly decreased expression of $p 27 k i p 1$ mRNA, suggesting an alternative mechanism of p27kip in these patients. In this study, patients with a smoking habit displayed methylation of $2 C D K I$ genes in their pancreatic carcinoma specimens. We concluded that epigenetic modification via hypermethylation represents a critical mechanism for the inactivation of $C D K I$ genes in pancreatic carcinoma.
\end{abstract}

\section{Introduction}

Pancreatic carcinoma is one of the leading causes of cancer mortality worldwide. Pancreatic cancer has been reported to be associated with various environmental and lifestyle risk

Correspondence to: Dr YingQi Zhou, The Third General Surgery Department, Zhanghai Hospital, Second Military Medical University, Shanghai, P.R. China

E-mail: 50803248@163.com

Key words: p15INK4b, p16INK4a, p21cip1, p27kip1, methylation, pancreatic carcinoma factors. Although the molecular etiology of pancreatic carcinoma is unclear, age and cigarette smoking are the unequivocal risk factors (1).

Due to the aggressive nature of the disease and the difficulties in diagnosis, the overall 5-year survival rate of pancreatic carcinoma is less than 5\% $(2,3)$. Novel approaches for the diagnosis and treatment of pancreatic cancer are necessary to improve the survival rate.

Cancer is a genetic disease where alterations in several genes accumulate and lead to a cancer cell growth advantage. Cell-cycle progression is driven by cyclins and cyclin-dependent kinases (CDKs). The activities of cyclin-CDK complexes are modulated by two classes of CDK inhibitor (CDKI) (4). The INK4 CDKI proteins (p15INK4, p16INK4, p18INK4 and p19INK4) sequester CDKs and inhibit the formation of CDK-cyclin complexes, whereas the Cip/Kip CDKIs (p21Waf1/ Cip1,p27Kip1 and p57Kip2) bind to cyclin-CDK complexes (5). p21 (also known as waf1, cip1 or sdi1) is an important cellular checkpoint molecule for the inhibition of a range of cyclin-CDK activities. In our previous study, we demonstrated that association of p21cip1 with CDK2/cyclin E blocks cell-cycle progression at multiple points $(6,7)$. The INK4A locus encodes two unrelated proteins, p14ARF and p16INK4a. p16INK4a is a specific inhibitor of the cyclin D-dependent kinases CDK4 and CDK6 (8) and antagonizes their ability to phosphorylate the retinoblastoma $\mathrm{Rb}$ ) family of proteins and so prevent exit from the G1 phase of the cell cycle (9). INK4A is important in mediating the signals that constrain the cell cycle in response to hyperproliferative signals, and, furthermore, are the most frequently inactivated tumor suppressor genes in human cancer $(10,11)$.

Promoter methylation is an alternative form of gene silencing, which relies on epigenetic factors. Previous reports have revealed that aberrant INK4a or Cip/Kip promoter methylation is a frequent event in human tumors (12). Studies have indicated that suppressed expression by aberrant promoter methylation may be an alternative mechanism for inactivation of the tumor suppressor gene in pancreatic cancer cases (13).

In the present study, we reported that reduced levels of p15INK4b, p16INK4a, p21cip1 and p27kip1 CDKI proteins and mRNA are prominent features of pancreatic carcinoma. We observed that promoter hypermethylation of genes was partly correlated with decreased p15INK4b, p16INK4a and p21cip1, but not $p 27 k i p 1$, mRNA in the tumors from clinical patients. 


\section{Materials and methods}

Study subjects. Five frozen fresh tumor specimens were surgically isolated from patients with pancreatic carcinoma. All of these patients were admitted into the third General Surgery Department, Zhongshan Hospital. Ethical approval for this study from the Zhongshan hospital and agreement by all patients were obtained.

Western blot analysis. The tumor and human normal tissues were prepared in lysis buffer from the MC-CelLytics kit (Shenergy Biocolor, Shanghai, China). The protein content was determined using the Bradford calorimetric assay method (Shenergy Biocolor). The lysate was resolved by $10 \%$ polyacrylamide-sodium lauryl sulfate gel electrophoresis and Immobilon-P transfer membrane (Millipore, MA, USA). Antibodies used for detection were p15INK4b (Santa Cruz), p16INK4a (Santa Cruz), p21cip1 (Cell Signaling) and p27kip1 (Santa Cruz). Then, the blot was incubated with a secondary antibody, IRDye 800 conjugated affinity purified anti-mouse or anti-rabbit IgG (Rockland Immunochemicals, Gilbertsville, PA, USA), and detected with an Odyssey Infrared Imaging System (LI-COR Bioscienceces, Lincoln, NE, USA).

Real-time RT-PCR analysis. Total RNA was extracted using TRIzol reagent (Invitrogen, Carlsbad, CA, USA) from tumor specimens and normal tissues. RNA was reverse transcribed using a PrimeScript ${ }^{\mathrm{TM}}$ RT reagent kit (DRR037A; Takara, Dalian, China). Real-time PCR analysis was performed in a final volume of $25 \mu \mathrm{l}$, containing $2 \mu \mathrm{l}$ of cDNA template, $0.5 \mu \mathrm{l}$ of each primer $(10 \mu \mathrm{M})$ and $12.5 \mu \mathrm{l}$ of a SYBR-Green master mix (2X) according to the instructions of the Real-time PCR kit (Takara, Japan) to evaluate the levels of mRNA expression. The following primers were used: 5'-AAGCTGAGCCCAGGT CTCCTA-3' (forward)and 5'-CCACCGTTGGCCGTAAACT-3' (reverse) for p15INK4b; 5'-ACCCTTGTGCCTCGCTCAG-3' (forward) and 5'-GGTCTGCCGCCGTTTTC-3' (reverse) for p21cip1, and published primers for p16INK4a (14) and p27kip1 (15). The average amount of the genes was normalized to the levels of GAPDH (16), an endogenous housekeeping gene.

Methylation analysis. The tumor and normal tissue DNA was extracted by Tissue Genomic Isolation kits (Dingguo Bio-tech, Beijing, China). For methylation-specific PCR (MSP), samples were prepared according to the instructions of the CpGenome ${ }^{\mathrm{TM}}$ FAST DNA Modification kit (S7824; Chemicon International, CA, USA). Bisulfite-treated DNA was amplified using MSP primers specific for either methylated or unmethylated DNA using published primers for p15INK4b (17), p16INK4a (17), p21cip1 (18) and p27kip1 (18). PCR was carried out with Ex-Taq Hot Start DNA polymerase (Takara). The annealing temperatures used were $60^{\circ} \mathrm{C}$ for $\mathrm{p} 15 \mathrm{U} / \mathrm{M}, 65 / 60^{\circ} \mathrm{C}$ for $\mathrm{p} 16$ $\mathrm{U} / \mathrm{M}, 57^{\circ} \mathrm{C}$ for $\mathrm{p} 21 \mathrm{U} / \mathrm{M}$ and $55^{\circ} \mathrm{C}$ for p27 U/M.

\section{Results}

Reduced levels of p15INK4b, p16INK4a,p21cipl and p27kipl proteins detected in pancreatic carcinoma. The events leading to pancreatic carcinoma development remain largely unknown.

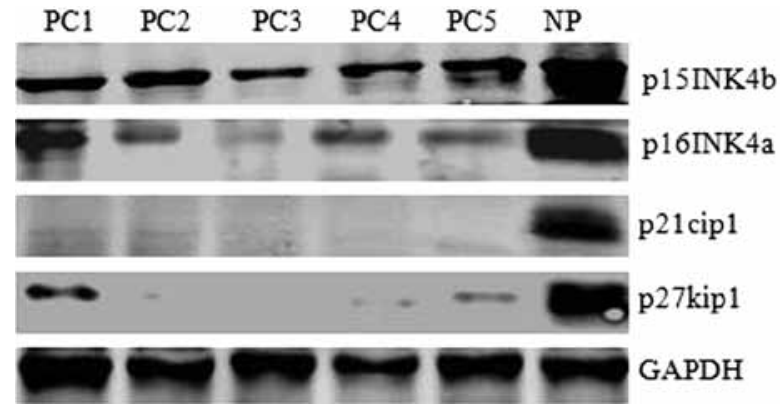

Figure 1. Reduced levels of p15INK4b, p16INK4a, p21cip1 and p27kip1 were detected in pancreatic carcinoma. Western blotting was performed to assess p15INK4b, p16INK4a, p21cip1 and p27kip1 protein levels in pancreatic carcinoma specimens (PC) and normal pancreatic tissue (NP) with GAPDH as the control.

Several studies have shown that the Rb tumor-suppressive pathway is abrogated in almost all studied cases of pancreatic carcinoma, and this disruption is caused exclusively by inactivation of CDKI (19). p15INK4b, p16INK4a, p21cip1 and p27kip1 are considered the most important CDKIs. This led us to ask whether these CDKIs are involved in pancreatic tumor formation. We examined p15INK4b, p16INK4a, p21cip1 and p27kip1 expression in 5 pancreatic carcinoma specimens and controls using western blotting. As shown in Fig. 1, p15INK4b, p16INK4a, p21cip1 and p27kip1 protein levels in 5 pancreatic carcinoma specimens (PC1-5) were much lower than in normal human pancreatic tissue (NP). These data suggest that reduced levels of p15INK4b, p16INK4a, p21cip1 and p27kip1, the negative regulators of cell progression, may be causative of tumor formation in pancreatic carcinoma.

Reduced levels of p15INK4b, p16INK4a, p21cipl and p27kipl mRNA expression detected in pancreatic carcinoma. Subsequently, we were interested in whether aberrant mRNA expression levels contributed to these CDKI alterations in pancreatic carcinoma specimens from patients. We evaluated p15INK4b,pl6INK4a,p21cipl and p27kipl mRNA by real-time RT-PCR analysis to determine whether decreased mRNA accumulation contributed to their protein levels. As shown in Fig. 2, it was preceded by a $>3$-fold reduction in $p 15 I N K 4 b$ mRNA expression, a $>4$-fold reduction in pl6INK 4 a mRNA expression, a $>3$-fold reduction in 21cipl mRNA expression and a $>4$-fold reduction in p27kipl mRNA expression of PC specimens, as compared to human NP tissue. Our findings suggested that the reduced levels of p15INK4b, p16INK4a, p21cip1 and p27kip1 proteins were likely due to a reduction in their mRNA synthesis, stability or translation in pancreatic carcinoma.

Methylation status of the p15INK4b, p16INK4a, p21cipl and p27kipl promoter region in pancreatic carcinoma. To explore the mechanism associated with the transcriptional silencing of the p15INK4b, p16INK4a,p21cipl and p27kipl genes, we examined 5 PC and human NP tissue samples to see whether there was methylation alteration in a $\mathrm{CpG}$-rich region of the transcription initiation site of the these genes. The presence or absence of methylation in the promoters of the p15INK4b,p16INK4a,p21cipl and p27kipl genes was determined by MSP. MSP distinguishes unmethylated from 

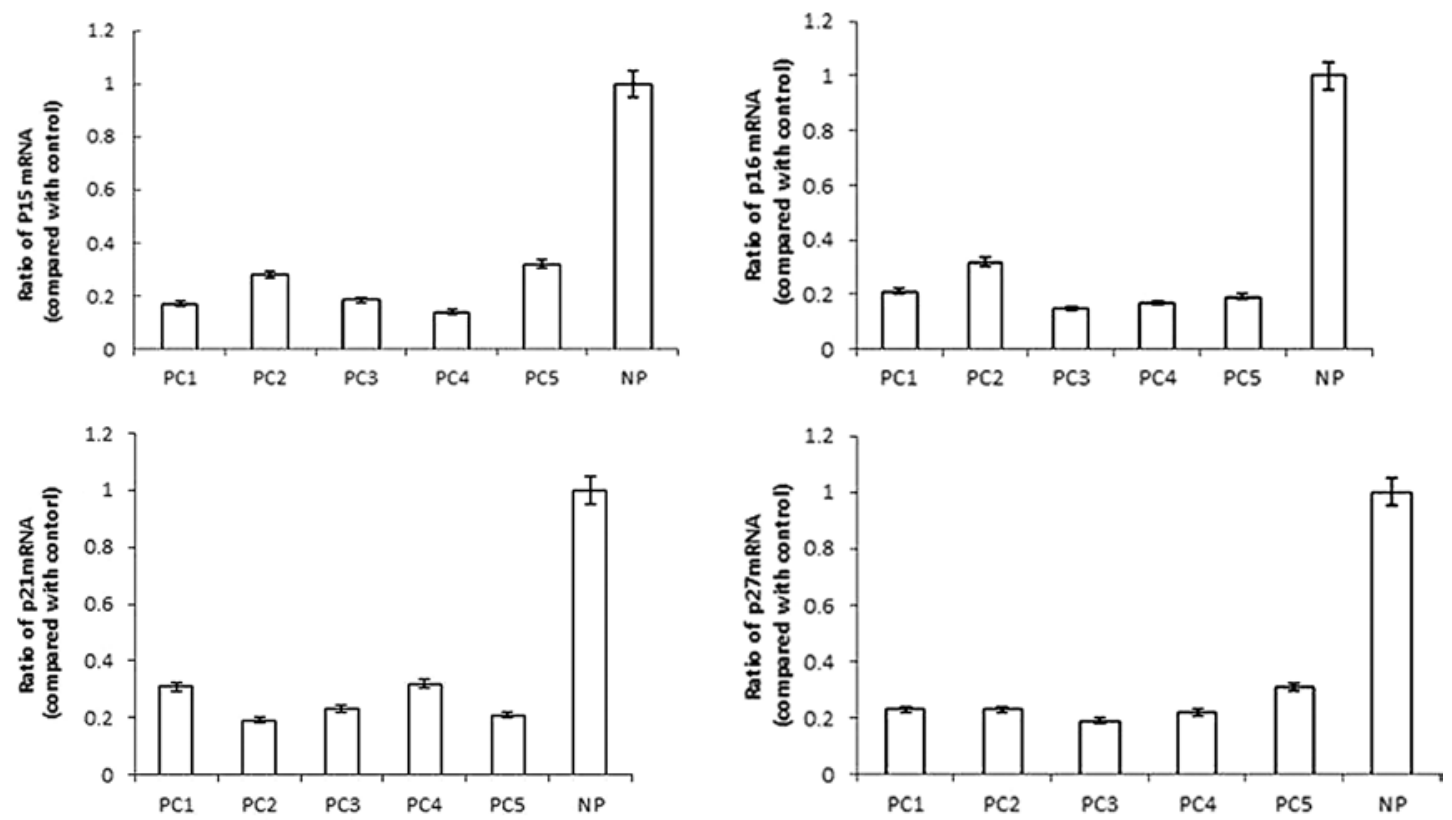

Figure 2. Reduced p15INK4b, p16INK4a, p21cip1 and p27kip1 mRNA expression was detected in pancreatic carcinoma. Real-time PCR was performed to assess p15INK4b, p16INK4a, p21cipl and p27kip1 mRNA in pancreatic carcinoma specimens (PC) and normal pancreatic tissue (NP) as the control, and normalized to GAPDH expression. The error bars indicate the standard error of the mean of 3 independent experiments.

$\begin{array}{lllllll}\mathrm{PC} 1 & \mathrm{PC} 2 & \mathrm{PC} 3 & \mathrm{PC} 4 & \mathrm{PC} 5 & \mathrm{NP}\end{array}$

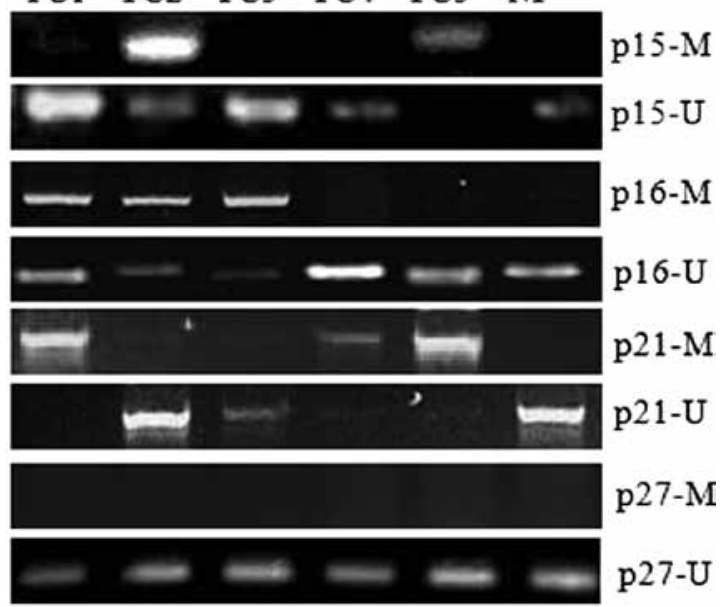

Figure 3. Methylation status of the $p 15 I N K 4 b, p 16 I N K 4 a, p 21$ cipl and p27kip1 promoter regions in pancreatic carcinoma. Methylation-specific PCR was carried out. The presence of a PCR product in lanes M indicates the presence of methylated gene promoter and the presence of a product in lanes $\mathrm{U}$ indicates the presence of an unmethylated gene promoter in pancreatic carcinoma specimens (PC) and normal pancreatic tissue (NP).

methylated alleles based on sequence changes produced following bisulfite treatment of DNA, which converts unmethylated cytosine to uracil, and subsequent PCR using primers designed for either methylated or unmethylated DNA. As shown in Fig. 3, promoter hypermethylation was detected in PC2 and PC5 samples in the $p 15 I N K 4 b$ gene, $\mathrm{PC} 1,2$ and 3 samples in the p16INK $4 a$ gene or PC1, 4 and 5 samples in the p21cipl gene, while no detectable promoter hypermethylation of the p27kipl gene was found in all 5 PC samples. No detectable promoter hypermethylation of the p15INK $4 b$, p16INK4a, p2lcipl and p27kipl genes was found in human
Table I. Association between smoking and methylation status.

\begin{tabular}{lcccccc}
\hline No. & Gender & $\begin{array}{c}\text { Age } \\
\text { (years) }\end{array}$ & $\begin{array}{c}\text { Cigarette } \\
\text { smoking }\end{array}$ & p15 & p16 & p21 \\
\hline 1 & M & 42 & Yes & $\times$ & $\sqrt{ }$ & $\sqrt{ }$ \\
2 & F & 62 & Yes & $\sqrt{ }$ & $\sqrt{ }$ & $\times$ \\
3 & F & 49 & No & $\times$ & $\sqrt{ }$ & $\times$ \\
4 & F & 60 & No & $\times$ & $\times$ & $\sqrt{ }$ \\
5 & M & 56 & Yes & $\sqrt{ }$ & $\times$ & $\sqrt{ }$ \\
\hline
\end{tabular}

${ }^{\mathrm{a}}$ Methylation status of the gene promoters.

NP tissue samples (Fig. 3). Thus, aberrant DNA promoter hypermethylation of the $p 15 I N K 4 b, p 16 I N K 4 a$ and $p 21 c i p l$ genes was thought to play a role in several cases of pancreatic carcinoma that had markedly decreased expression of p15INK4b, p16INK4a and p2lcipl mRNA, concomitant with loss of p15INK4b, p16INK4a and p21cip1 proteins, but not p27kip1 protein (Figs. 2 and 3). Thus, we suggest that DNA hypermethylation associated with transcriptional silencing of the p15INK4b, p16INK4a and p21cipl genes may partly contribute to pancreatic carcinoma progression.

Pancreatic carcinoma patients with smoking habits displayed methylation of 2 CDKI genes. According to our results, pancreatic carcinoma patients showed reduced levels of p15, p16, p21 and p27 mRNA or proteins. To investigate the correlation between smoking habit and the methylation status of the promoter region in pancreatic carcinoma, these 5 randomly selected pancreatic carcinomas removed from the patients were further studied. As shown in Table I, the patients having smoked for $>15$ years (PC1, 2 and 5) displayed methylation of 
2 genes in their pancreatic carcinoma specimens. On the other hand, the other 2 pancreatic carcinoma specimens (PC3 and 4), with only 1 methylation region in the $C D K I$ genes, occurred in the patients without a cigarette smoking record (Table I). Thus, we suggest that cigarette smoking may be associated with pancreatic tumorigenesis by inducing the methylation of the promoter regions of the CDKI genes.

\section{Discussion}

In this study, we revealed that the reduced levels of two classes of CDKI protein, including p15INK4b, p16INK4a, p21cip1 and p27kip1, are a prominent feature of pancreatic carcinomas. Moreover, a reduced amount of $p 15 I N K 4 b, p 16 I N K 4 a, p 21 c i p 1$ and $27 \mathrm{kipl} \mathrm{mRNA}$ expression was found in all of the pancreatic carcinoma samples. We observed that hypermethylation of the p15INK4b, p16INK4a and p21cipl promoters, but not the p27kipl promoter, was partly correlated with markedly decreased mRNA expression. Thus, we suggest that hypermethylation associated with transcriptional silencing of the pl5INK4b, p16INK4a and p21cipl gene may contribute to the progression of certain pancreatic carcinomas.

Due to the few treatment options for pancreatic carcinoma, understanding of the molecular pathology is a prerequisite for identifying potential molecular targets for drug therapy. CDKIs are negative regulators of cell-cycle progression at the G1-S restriction point. In this study, we showed that the reduced levels of p15INK4b, p16INK4a, p21cip1 and p27kip1 proteins were a prominent feature in the 5 pancreatic carcinoma specimens analyzed (Fig. 1), indicating that the reduced levels of p15INK4b, p16INK4a, p21cip1 and p27kip1, the negative regulators of cell progression, are involved in the tumor formation in pancreatic carcinoma.

Moreover, a reduced amount of p15INK4b, p16INK4a, p21cipl and p27kipl mRNA normal expression was found in 5 pancreatic carcinoma samples (Fig. 2). We observed patients with a markedly decreased $>3$-fold reduction in p15INK $4 b$ mRNA, a 4-fold reduction in pl6INK4a mRNA, a 3-fold reduction in p21cipl mRNA and a 4-fold reduction in p27kipl mRNA, concomitant with reduced levels of these CDKI proteins in sporadic pancreatic carcinoma (Figs. 1 and 2). The methylation status of the p15INK4b, p16INK4a, p21cipl and p27kipl promoter region was detected using MSP. The DNA hypermethylation promoter was found in $40 \%$ ( 2 of 5 ) of the p15INK $4 b$ genes, $60 \%$ (3 of 5) of the pl6INK4a genes, $60 \%$ (3 of 5) of the p21cipl genes and $0 \%$ (0 of 5) of the p27kipl genes, and markedly correlated with decreased mRNA expression in pancreatic carcinoma patients (Figs. 2 and 3). Moreover, all 5 pancreatic carcinoma patients $(100 \% ; 5 / 5)$ displayed methylation of 1 or more genes, $60 \%$ (3/5) displayed methylation of 2 genes, while control tissue did not display any methylation. It has been shown that aberrant methylation is the most prominent feature of pancreatic carcinoma, causing alterations in the expressions of genes (13). Thus, we demonstrated that the epigenetic modification of $p 15 I N K 4 b, p 16 I N K 4 a$ and $p 21 c i p 1$ via hypermethylation represents a critical mechanism for, at least in part, the inactivation of these genes in pancreatic carcinoma. In all 5 of our pancreatic carcinoma patients with markedly decreased expression of p27kipl mRNA, we were unable to detect hypermethylation in the promoter region, suggesting an alternative mechanism of the p27kipl gene in these patients.

Pancreatic carcinoma has been reported to be associated with various environmental and lifestyle risk factors, occupational exposures and medical conditions; however, the only risk factors consistently reported are age and smoking status, and the etiology of the disease remains largely unknown (1). In this study, we analyzed the correlation between smoking status and biological events in 5 randomly selected sporadic pancreatic carcinomas. Indeed, the patients smoking cigarettes for more than 15 years (PC1, 2 and 5) displayed methylation of 2 genes in their pancreatic carcinoma specimens. On the other hand, the other 2 pancreatic carcinoma specimens (PC3 and 4) with only 1 methylation region in the $C D K I$ genes were surgically isolated from the patients without a cigarette smoking record (Table I). Thus, we suggest that cigarette smoking may be associated with pancreatic tumorigenesis by inducing the methylation of the promoter regions of the CDKI genes.

Our previous data suggested that expression of p21cip1 stops cell growth progression by inactivation of CDK activity, which in turn blocks the cell cycle at the G1 and G2 phases $(20,21)$. Consistent with our previous finding that heightened CDK/cyclin signal transduction concomitant with loss of p27kip1 (22), the present study indicates that the reduced levels of the CDKI protein are a prominent feature of pancreatic carcinoma. These data suggest that the reduced levels of p15INK4b, p16INK4a, p21cip1 and p27kip1 may lead to defects in cell-cycle regulation and confer a selective growth advantage for pancreatic cancer cells. Thus, we showed that reduced levels of p15INK4b, p16INK4a, p21cip1 and p27kip1, the negative regulators of cell progression, may be causative of tumor formation in pancreatic cancer cells.

Taken together, reduced levels of p15INK4b, p16INK4a, p21cip1 and p27kip1 are a fundamental event in tumor formation in the clinical pancreatic carcinoma patients. The CDK inhibitors may interact to mediate signals that are critical growth inhibitors. This growth regulatory circuit would be disrupted, such as in pancreatic carcinoma. Thus, our observations have significant implications for understanding the importance of p15INK4b, p16INK4a, p21cip1 and p27kip1; reduced levels of these CDKI proteins contribute to tumorigenesis in pancreatic carcinoma and developing ways to target aberrantly active parts of this growth regulatory pathway may lead to increased survival.

\section{Acknowledgements}

This study was supported by the Natural Science Foundation of China (81071740) and the Shanghai Science Foundation (10ZR1406300).

\section{References}

1. Ghadirian P, Lynch HT and Krewski D: Epidemiology of pancreatic cancer: an overview. Cancer Detect Prev 27: 87-93, 2003.

2. Hezel AF, Kimmelman AC, Stanger BZ, Bardeesy N and Depinho RA: Genetics and biology of pancreatic ductal adenocarcinoma. Genes Dev 20: 1218-1249, 2006.

3. Jemal A, Siegel R, Ward E, et al: Cancer statistics. CA Cancer J Clin 58: 71-96, 2008.

4. Elledge SJ, Winston J and Harper JW: A question of balance: the role of cyclin-kinase inhibitors in development and tumorigenesis. Trends Cell Biol 6: 388-392, 1996. 
5. Harper JW, Adami GR, Wei N, Keyomarsi K and Elledge SJ: The $\mathrm{p} 21 \mathrm{Cdk}$-interacting protein Cip1 is a potent inhibitor of G1 cyclin-dependent kinases. Cell 75: 805-816, 1993.

6. Tang JJ, Shen C and Lu YJ: Requirement for pre-existing of p21 to prevent doxorubicin-induced apoptosis through inhibition of caspase-3 activation. Mol Cell Biochem 291: 139-144, 2006.

7. Lu Y, Yamagishi N, Yagi T and Takebe H: Mutated p21(WAF1/ CIP1/SDI1) lacking CDK-inhibitory activity fails to prevent apoptosis in human colorectal carcinoma cells. Oncogene 16: 705-712, 1998 .

8. Serrano M, Hannon GJ and Beach D: A new regulatory motif in cell-cycle control causing specific inhibition of cyclin D/CDK4. Nature 366: 704-707, 1993.

9. Sherr CJ and Roberts JM: CDK inhibitors: positive and negative regulators of G1-phase progression. Genes Dev 13: 1501-1512, 1999.

10. Hainaut P, Soussi T, Shomer B, et al: Database of p53 gene somatic mutations in human tumors and cell lines: updated compilation and future prospects. Nucleic Acids Res 25: 151-157, 1997.

11. Hall M and Peters G: Genetic alterations of cyclins, cyclindependent kinases, and Cdk inhibitors in human cancer. Adv Cancer Res 68: 67-108, 1996.

12. Perrone F, Tabano S, Colombo F, et al: p15INK4b, p14ARF, and p16INK4a inactivation in sporadic and neurofibromatosis type 1-related malignant peripheral nerve sheath tumors. Clin Cancer Res 9: 4132-4138, 2003.

13. Klump B, Hsieh CJ, Nehls O, et al: Methylation status of p14ARF and p16INK4a as detected in pancreatic secretions. Br J Cancer 88: 217-222, 2003.
14. Kurihara Y, Egawa K, Kunimoto S, Takeuchi T and Nose K: Induction of p16/INK4a gene expression and cellular senescence by toyocamycin. Bio Pharm Bull 25: 1272-1276, 2002.

15. Cen B, Li H and Weinstein IB: Histidine triad nucleotide-binding protein 1 up-regulates cellular levels of p27KIP1 by targeting ScfSKP2 ubiquitin ligase and Src. J Biol Chem 284: 5265-5276, 2009.

16. West AB, Kapatos G, O'Farrell C, et al: $\mathrm{N}$-myc regulates parkin expression. J Biol Chem 279: 28896-28902, 2004.

17. Herman JG, Graff JR, Myöhänen S, Nelkin BD and Baylin SB: Methylation-specific PCR: a novel PCR assay for methylation status of CpG islands. Proc Natl Acad Sci USA 93: 9821-9826, 1996.

18. Brakensiek K, Länger F, Kreipe H and Lehmann U: Absence of p21CIP1, p27KIP1 and p57KIP2 methylation in MDS and AML. Leuk Res 29: 1357-1360, 2005.

19. Schneider G and Schmid RM: Genetic alterations in pancreatic carcinoma. Mol Cancer 22: 15, 2003.

20. Wu H, Chen Y, Wang ZY, et al: Involvement of 21 (waf1) in merlin deficient sporadic vestibular schwannomas. Neuroscience 170: 149-155, 2010.

21. Ji Y, Ma SL, Zhang YP, et al: A combined treatment TNF- $\alpha /$ Gefitinib alleviates the resistance to Gefitinib in PC-9 cells with acquired resistance to Gefitinib. Anticancer Drug 20: 832-837, 2009.

22. Wang Z, Lu Y, Tang J, Wang $\mathrm{H}$ and $\mathrm{Wu} \mathrm{H}$ : The phosphorylation status of merlin in sporadic vestibular Schwannomas. Mol Cell Biochem 324: 201-206, 2009. 